\title{
LA INTERPRETACIÓN ASISTENCIAL SANITARIA EN ITALIA Y ESPAÑA DESDE UN PUNTO DE VISTA INTERDISCIPLINAR. UN ESTUDIO POBLACIONAL SOBRE LAS DOS REALIDADES MULTICULTURALES \\ Valentina Racioppi \\ Universidad de Málaga
}

\section{ABSTRACT}

In this article we present a field study of the resident and visitor foreign population in Italy and Spain. The aim of this study is to determine if the presence of a professional healthcare interpreter is really necessary, since both countries show a lack of regularization from their institutions. Nevertheless, Spain has a greater awareness and development of this branch of interpreting from the academic point of view. With this population study we aim to show real data to encourage once more the institutions and universities to cooperate in order to train and regularize the professional role of the healthcare interpreter.

KEY WORDS: Healthcare Interpreting, Public Services, Healthcare Services, Multiculturalism, Population Study.

\section{RESUMEN}

En este artículo se presenta un estudio de campo sobre la población extranjera residente y flotante en Italia y España, con el cual se quiere comprobar la real necesidad de la figura profesional del intérprete asistencial sanitario. De hecho, aunque en España existe una mayor consciencia y desarrollo a nivel académico de esta rama de la interpretación, en ambo países sigue una falta de regularización a nivel institucional. Con este estudio poblacional queremos exhibir datos ciertos con los cuales se anime una vez más las instituciones y las universidades a colaborar para formación y regularización de la figura profesional del intérprete sanitario.

PALABRAS CLAVE: interpretación asistencial sanitaria, servicios públicos, servicios sanitarios, multiculturalidad, estudio poblacional.

FECHA DE RECEPCIÓN: 25/02/2015

FECHA DE ACEPTACIÓN: 29/06/2015

PÁGINAS: 635-656 


\section{INTRODUCCIÓN}

Nuestro trabajo trata de analizar la situación de la interpretación asistencial sanitaria en Italia y España desde una perspectiva interdisciplinar, en concreto a través de un estudio poblacional a nivel nacional de estas dos realidades. El objetivo de este trabajo es, por lo tanto, presentar una panorámica general y actual sobre la situación migratoria y multicultural de estos dos países y proponer una visión contrastiva que confirme o desmienta la necesidad de esta rama profesional tanto en Italia como en España.

La sociedad actual se caracteriza por una multietnicidad y multiculturalidad en continuo crecimiento las cuales, debidas a los persistentes fenómenos de emigración, que no se pueden interpretar de manera provisional, sino que requieren nuestra constante atención. Es cierto, dentro de un contexto multicultural como el de la Unión Europea, la inmigración se considera el cuarto asunto más importante que afecta a los europeos (Comisión Europea 2006: 26).

Tanto en Italia como en España, la introducción de poblaciones extranjeras exige el empleo de una perspectiva de integración cultural y de la consiguiente activación de "profundas transformaciones culturales en los hábitos sociales» (Fumagalli en Falvo 2010: 123). Entre otras, se ha destacado en los últimos años la necesidad entre las instituciones de mediar lingüística y culturalmente con el ciudadano extranjero, reduciendo la distancia en los derechos y deberes entre la población inmigrante y la nativa. Si partimos de esta observación, consideramos fundamental investigar sobre el factor inmigración en España y en Italia y comparar estas dos realidades para llevar a cabo nuestro estudio contrastivo sobre el papel y la relevancia de la interpretación sanitaria.

\section{TIPOLOGÍA DE DATOS Y ESTRATEGIA DE EXTRACCIÓN}

Para nuestra recopilación de datos, hemos consultado como fuentes primarias el Instituto Nacional de Estadística Español INE y el Instituto Central de Estadística ISTAT para los datos nacionales sobre Italia; también, hemos extraído datos del Instituto de Estudios Turísticos IET relativos al turismo extranjero en España y hemos obtenido información a escala internacional de las fuentes estadísticas de la OCDE (Organización para la Cooperación y el Desarrollo Económicos) y de la oficina europea de estadística Eurostat, sobre todo por lo que se refiere a datos laborales.

Nuestra recopilación se realizó a través de un recorrido preciso, que empezó con fuentes relativas a Italia y que se concluyó con España, para establecer primero una visión completa de ambos países y luego presentar una 
comparación más lógica y completa de estas dos realidades. En concreto, hemos partido de un enfoque más general sobre las poblaciones extranjeras más presentes en Italia. Una vez establecido cuáles son las nacionalidades y lenguas extranjeras más representadas, hemos centrado nuestra atención en el estudio de las razones que llevan a estos extranjeros a migrar al bel paese y cuáles son las actividades profesionales a las que más se dedican en el país de llegada.

Por lo que atañe a los datos poblacionales a escala nacional, hemos decidido analizar los datos del último bienio disponible para enseñar también el dinamismo que estos valores conllevan, mientras que para los datos laborales, hemos ahondado nuestra búsqueda incluyendo valores a escala mundial que nos han ofrecido un informe detallado y actual sobre el tema en ambos países. El paso siguiente consistió en encontrar datos más específicos sobre los colectivos inmigrantes y la asistencia sanitaria. Con respecto a Italia, pudimos localizar tanto datos relativos a las principales enfermedades que afectan a este sector de la población como a los problemas lingüísticos más representativos de la comunicación médico-paciente.

Por último, teniendo en cuenta que Italia y España son ambos destinos turísticos por excelencia, hemos decidido dedicar un pequeño apartado a los turistas extranjeros, que también se pueden beneficiar de los servicios sanitarios.

\section{ESTUDIO DE POBLACIÓN: LOS EXTRANJEROS EN ITALIA}

Tras un siglo caracterizado por una fuerte emigración, sobre todo a EE. UU., Argentina, Brasil y Australia, a partir de los años setenta del siglo XX, Italia empezó a acoger un número siempre progresivo de extranjeros. Este flujo migratorio se debió, por un lado, a las ventajas que acuerdos bilaterales y tratados ofrecían a los descendentes de emigrados italianos, como el Convenio de Doble Nacionalidad de 1971 entre la República Argentina y la República Italiana o el Tratado de Maastricht de 1992 sobre la integración europea (Zanin y Mattiazzi 2011: 150). Por otro lado, ha sido el resultado de hechos económicos y sociales, entre otros los más recientes refugiados por la Primavera Árabe, que clasificaron a Italia cuarto país de recepción en 2011 (OECD 2014a: 136).

Como consta el informe sobre la inmigración en Italia del OECD (2014b), en la última década el número de inmigrantes se ha triplicado. Algo análogo ha ocurrido en España. Según los datos del ISTAT de 2014, en el territorio italiano las nacionalidades extranjeras más representadas en 2013 eran Rumanía (933 354), Albania (464 962), Marruecos (426 791), China (223 367), Ucrania (191 725) y Filipinas (139 734). Hay que precisar que un informe 
ISTAT más reciente sobre los ciudadanos no comunitarios $^{1}$ registra Marruecos como el segundo país extranjero más presente en Italia (524 775), seguido por Albania (502 546), China (320 794), Ucrania (233 726) y Filipinas (165 783). Juntos, estos países no comunitarios representan casi la mitad de la población no comunitaria total presente en Italia.
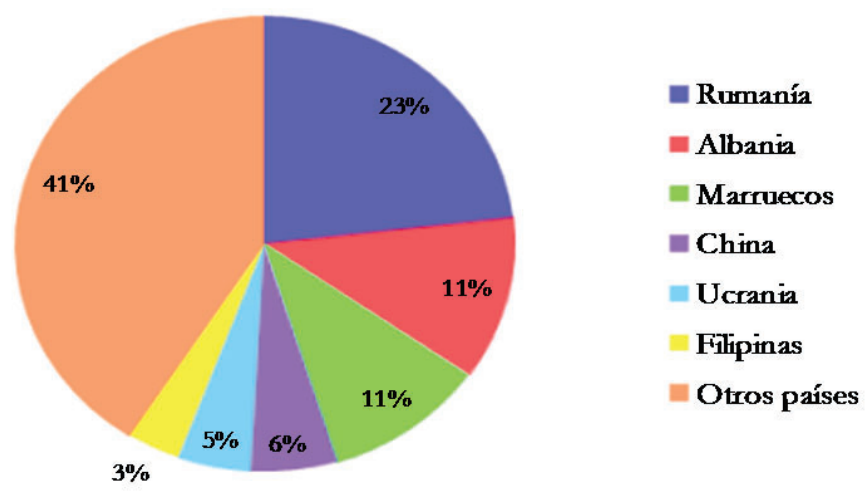

Gráfico 1. Principales poblaciones extranjeras presentes en Italia

Si pasamos a un análisis más localizado, podemos constar que la población extranjera residente no se distribuye de manera homogénea dentro de la península, sino que la gran mayoría de los inmigrantes prefiere el norte de Italia, con un 35,2\% repartido en el área oeste y el 26,6\% en el este. El resto de población extranjera se reparte en el $24,2 \%$ en el centro, el $10 \%$ en el sur, y solo el $4 \%$ en las islas. Finalmente, de los valores extraídos por cada región destacan Lombardía con el 23,1 \% de extranjeros residentes, Emilia-Romaña $(10,93 \%)$, Véneto $(10,90 \%)$ y Lacio $(10,68 \%)$, seguidas por Piamonte $(8,61 \%)$ y Toscana $(7,85 \%)$.

\begin{tabular}{|l|c|c|c|c|c|c|}
\hline \multirow{2}{*}{ Área } & \multicolumn{2}{|c}{ Italianos } & \multicolumn{2}{c}{ Extranjeros } & \multicolumn{2}{c|}{ Total } \\
\hline Noroeste & $\mathbf{2 0 1 3}$ & $\mathbf{2 0 1 4}$ & $\mathbf{2 0 1 3}$ & $\mathbf{2 0 1 4}$ & $\mathbf{2 0 1 3}$ & 2014 \\
\hline & 14339096 & 14428329 & 1426471 & 1702396 & 15765567 & 16130725 \\
\hline \multirow{2}{*}{ Noreste } & 10356462 & 10401367 & 1091343 & 1253119 & 11447805 & 11654486 \\
\hline
\end{tabular}

${ }^{1}$ Report ISTAT Cittadini non comunitari regolarmente presenti del 5 de agosto de 2014. 
Entreculturas 7-8 (enero 2016) ISSN: 1989-5097 Valentina Racioppi

\begin{tabular}{|c|c|c|c|c|c|c|}
\hline Centro & 10632199 & 10821012 & 968476 & 1249830 & 11600675 & 12070842 \\
\hline Sur & 13591781 & 13655646 & 385650 & 512173 & 13977431 & 14167819 \\
\hline Islas & 6486579 & 6554229 & 155687 & 204567 & 6642266 & 6758796 \\
\hline Italia & 55406117 & 55860583 & 4027627 & 4922085 & 59433744 & 60782668 \\
\hline
\end{tabular}

Tabla 1. Resumen de datos sobre la población en Italia. Clasificación por área total y parcial

Podemos justificar esta repartición si consideramos la economía de estas áreas; de hecho, el norte y el centro de Italia siguen siendo las áreas más prolíficas a nivel laboral (Ministero del Lavoro e delle Politiche Sociali 2014: 111).

Otra referencia que justifica este reparto la podemos encontrar en las razones que llevan a los extranjeros a migrar a Italia. En el siguiente gráfico se recoge un reparto aproximado de ciudadanos extranjeros entre 2012 y 2013 según los motivos que justificaron la emigración a Italia²

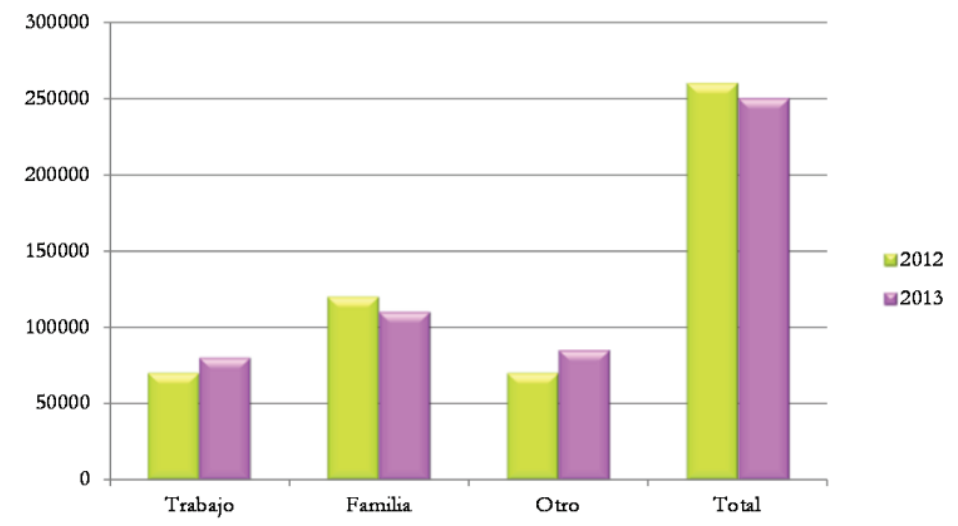

Gráfico 2. Razón de entrada de los ciudadanos no comunitarios en 2012 y 2013 (véase en página anterior)

Como se puede observar, aunque la familia sigue siendo la primera razón de la inmigración, se percibe un aumento en las razones laborales; también se nota un aumento en las motivaciones de estudio, asilo, petición de asilo y motivaciones humanitarias entre otras, que se agrupan en el valor "otro".

2Información obtenida de las elaboraciones del ISTAT sobre la base de datos del Ministerio del Interior italiano. 


\subsection{LOS EXTRANJEROS Y SUS PROFESIONES EN ITALIA}

Resulta muy interesante investigar sobre las categorías laborales relativas a los ciudadanos extranjeros en Italia, ya que son datos nos permiten obtener información sobre su nivel de especialización y su repartición en el territorio, permitiéndonos delimitar aún más las tipologías de los inmigrantes residentes en la península.

Podemos constar la relevancia de este tipo de información también en el documento elaborado en 2013 por el Centro Studi CNA³ L'imprenditoria straniera in Italia nel 2012, donde se afirma que dentro del marco europeo la importancia de la ocupación extranjera de Italia y España se clasifica respectivamente al segundo y primer lugar con porcentajes del 10,3\% y del 12,7\% (2013: 27).

Además, en el Informe sobre las Migraciones en el Mundo 2013, la Organización Internacional para las Migraciones (OIM) afirma que la mayoría de los migrantes son "trabajadores poco cualificados, aunque se dispone de muy poca información actualizada y fiable" (2013: 72). A este respecto, según la base de datos de la DIOC-E los migrantes que viven en los países de la OECD resultan repartidos en poco cualificados (44\%), con competencias intermedias (33\%) y altamente cualificados (22\%) (Dumont et al. 2010).

En el caso específico de Italia, hemos profundizado en el estudio proponiendo una tabla sobre la distribución de los extranjeros por tipología de trabajo según el área geográfica dentro del país. Hemos optado por centrarnos en cuatro macro-grupos profesionales según la clasificación redactada por el ISTAT en La classificazione delle professioni (2013).

De la tabla que proponemos a continuación observamos una lógica en la elección del área geográfica por parte del ciudadano extranjero. De hecho, observamos que el norte presenta el porcentaje más alto de "profesiones cualificadas", macro-grupo al que pertenecen, según el ISTAT, las categorías de los "juristas, altos funcionarios y empresarios", las "profesiones intelectuales, científicas y de alta especialización" y las "profesiones técnicas". Siempre en esta área observamos una concentración más alta de "obreros y artesanos", que comprenden los "artesanos, obreros especializados y agricultores" y los "conductores de plantas y obreros semi-cualificados de máquinas fijas y móviles"; al norte sigue el centro con el 35,5\% y el sur con el 20,5\%. En cambio, resulta más homogénea la distribución entre las tres áreas geográficas del macro-grupo "empleados y encargados de comercio y del sector servicios",

${ }^{3}$ Confederación Nacional de la Artesanía y de la Pequeña y Mediana Empresa [traducción literal]. 
el cual engloba los "empleados y las profesiones cualificadas en las actividades comerciales y en los servicios". Finalmente, es interesante observar que el sur casi dobla el norte con respecto a las "profesiones no cualificadas" con su $58 \%$.

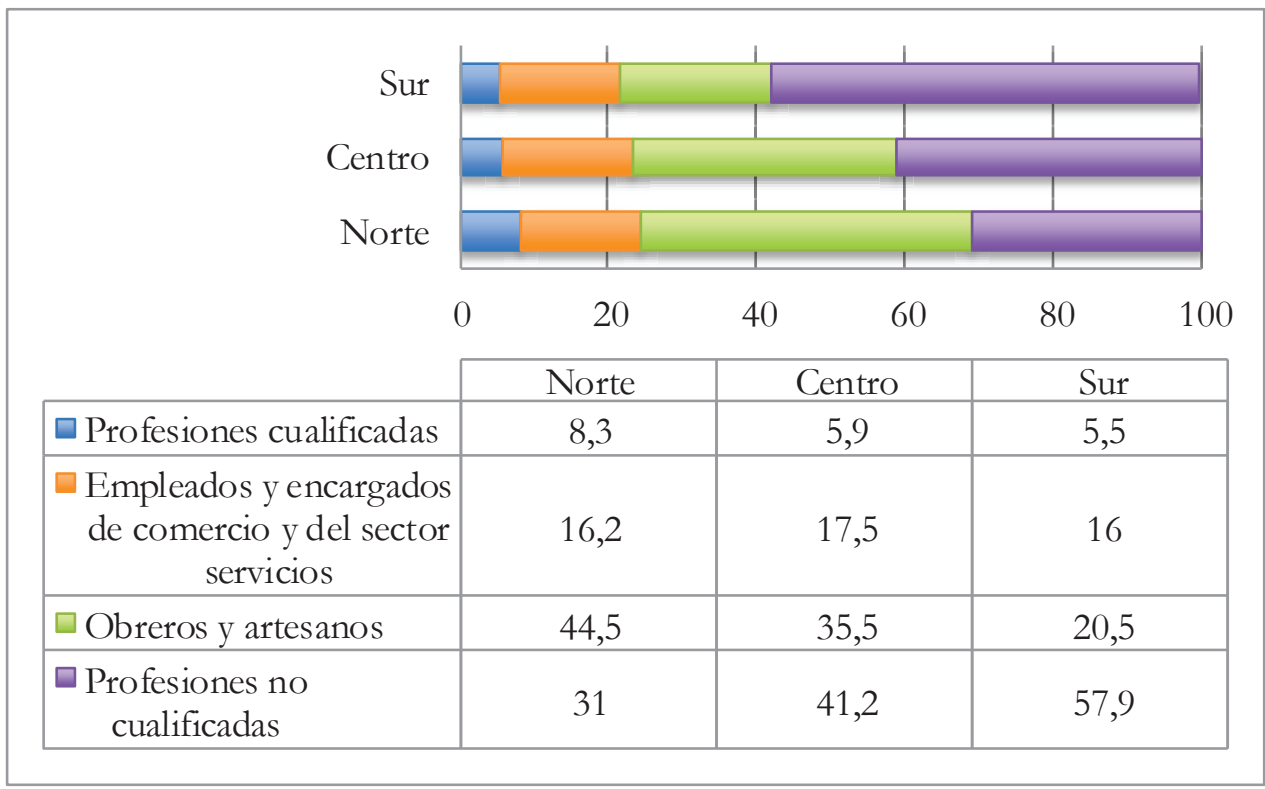

Tabla 2. Empleados extranjeros por profesión y distribución geográfica ${ }^{4}$

\subsection{LOS EXTRANJEROS RESIDENTES Y LOS SERVICIOS SANITARIOS}

A este punto, es preciso centrar nuestra atención en los pocos pero imprescindibles datos relativos a los extranjeros y los servicios sanitarios, ya que como veremos los datos socio-económicos que acabamos de analizar se reflejan en las problemáticas lingüísticas que atañen a los extranjeros a la hora de comunicarse con el personal médico.

Puesto que se trata de un estudio que tiene a que ver con la interpretación asistencial sanitaria, nos parece preciso empezar con identificar las enfermedades que más afectan a los extranjeros en Italia; de esta información se pueden desprender componentes valiosos tanto en el ámbito profesional como en el formativo de intérpretes y traductores, como por ejemplo la especialidad de lenguaje médico.

\footnotetext{
${ }^{4}$ Los valores de la tabla son porcentajes.
} 
Con respecto a las enfermedades, hemos identificado algunos de los sectores que más afectan a los inmigrantes. Según el ISTAT (2014), se trata sobre todo de enfermedades relacionadas con el aparato respiratorio $(6,54 \%)$, con el aparato digestivo y los dientes $(2,02 \%)$, con el sistema nervioso $(1,98 \%)$ y con el sistema musculoesquelético $(1,55 \%)$.

Acerca de la tipología de los servicios sanitarios, se destaca el empleo del servicio de urgencia tanto por parte de los inmigrantes comunitarios como de los no comunitarios, principalmente en el norte de Italia. A la urgencia siguen el servicio de ingreso, el médico de familia y el servicio de guardia médica5 ${ }^{5}$, este último más disfrutado en el sur de Italia (2014a: 9-10).

A la hora de acceder a los servicios sanitarios, la limitación lingüística puede constituir un serio problema para el extranjero. Un reciente estudio del ISTAT (2014a) releva que entre los inmigrantes de 14 años en adelante, el $13,8 \%$ tiene problemas en explicar en italiano los síntomas al médico y el $14,9 \%$ encuentra dificultades en comprender al personal sanitario.

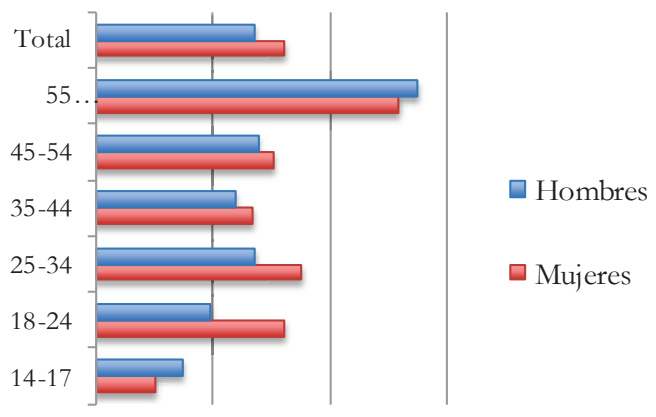

\section{Gráfico 3. Dificultades en entender al médico por edad y género ${ }^{6}$}

Como se puede observar en el gráfico 3, el problema lingüístico destaca además una diferencia de género, siendo las mujeres las que tienen más dificultades para expresarse o para entender al personal médico. También la edad es un factor que influye en la interacción paciente-médico; de hecho, una cuarta parte de los extranjeros de edad superior a los 54 tiene dificultad por el

\footnotetext{
${ }^{5}$ Es necesario precisar que el servicio de guardia médica italiano no corresponde al español. Con este término en Italia se define un servicio de continuidad asistencial que garantiza la asistencia de un médico de cabecera o un pediatra en las situaciones de emergencias durante las horas no laborales o durante los días festivo y de víspera.
}

${ }^{6}$ Datos del bienio 2011-2012. 
$25 \%$ en expresarse con el personal sanitario y por el $26,4 \%$ en comprenderlo (ISTAT 2014: 12).

Si pasamos en examinar las dificultades lingüística según la procedencia de los inmigrantes notamos cómo los ciudadanos extracomunitarios a la Unión Europea encuentran muchos más problemas en comunicar con el personal médico $(19 \%)$, mientras que los extranjeros de los Estados miembros con estas dificultades resultan ser el 5,6\%. Como era previsible, con respecto a las lenguas, observamos homogeneidad entre los inmigrantes procedentes de la UE, mientras que entre los extranjeros no comunitarios destacan los chinos $(43,3 \%)$, resultado que se explica por ser, el mandarín y el italiano, dos idiomas contrapuestos a nivel lingüístico.

\begin{tabular}{|c|c|c|c|c|c|c|}
\hline \multirow{2}{*}{ CIUDADANÍA } & \multicolumn{3}{|c|}{$\begin{array}{l}\text { EXPLICAR LOS SÍNTOMAS AL } \\
\text { MÉDICO }\end{array}$} & \multicolumn{3}{|c|}{ ENTENDER AL MÉDICO } \\
\hline & HOMBRES & MUJERES & MEDIA & HOMBRES & MUJERES & MEDIA \\
\hline PAÍSES UE & 4,9 & 5,8 & 5,4 & 5,9 & 5,8 & 5,8 \\
\hline Rumanía & 4,9 & 5,9 & 5,4 & 5,9 & 6,1 & 5,9 \\
\hline Polonia & 3,5 & 3,5 & 3,5 & 3,7 & 5,1 & 4,7 \\
\hline PAÍSES NO UE & 15,4 & 21,7 & 18,6 & 17,2 & 22,6 & 20,0 \\
\hline China & 46,1 & 46,5 & 46,3 & 49,6 & 47,8 & 48,7 \\
\hline India & 28,1 & 54,1 & 39,4 & 29,7 & 55,5 & 41,0 \\
\hline Filipinas & 26,1 & 30,9 & 28,9 & 38,5 & 33,1 & 35,3 \\
\hline Marruecos & 12,6 & 32,2 & 22,0 & 14,3 & 34,2 & 23,9 \\
\hline Túnez & 3,5 & 33,3 & 14,3 & 7,1 & 36,8 & 17,9 \\
\hline Albania & 7,9 & 16,9 & 12,0 & 9,0 & 14,5 & 11,5 \\
\hline Ucrania & 9,8 & 5,6 & 6,3 & 14,4 & 6,3 & 7,6 \\
\hline Moldavia & 6,5 & 3,5 & 4,4 & 7,7 & 5,7 & 6,3 \\
\hline TOTAL & 12,7 & 16,6 & 14,8 & 14,3 & 17,3 & 15,9 \\
\hline
\end{tabular}

Tabla 3. Dificultades lingüísticas en la interacción paciente-médico por idioma

Finalmente, hay que recordar que los problemas de comunicación entre el paciente extranjero y el personal sanitario dependen también del nivel de educación y del tiempo de estancia. De hecho, se registran una dificultad mayor entre los extranjeros más débiles a nivel socio-económico y que tienen un nivel 
de educación bajo. Según ISTAT (2014a) esta condición afecta principalmente a los extranjeros mayores de 45 años, con un porcentaje del 38,4\%. Sin embargo, es interesante notar que casi el $11 \%$ de los extranjeros que residen en Italia de más de diez años siguen teniendo problemas lingüísticos en este tipo de interacción. Este porcentaje puede encontrar una justificación si tenemos en cuenta el tipo de generación y la época en la que estos extranjeros migraron en la península itálica, ya que también la cultura y la mentalidades cambian según la generación y por consiguiente la necesidad o voluntad de aprender más o menos bien el idioma del nuevo país que nos acoge.

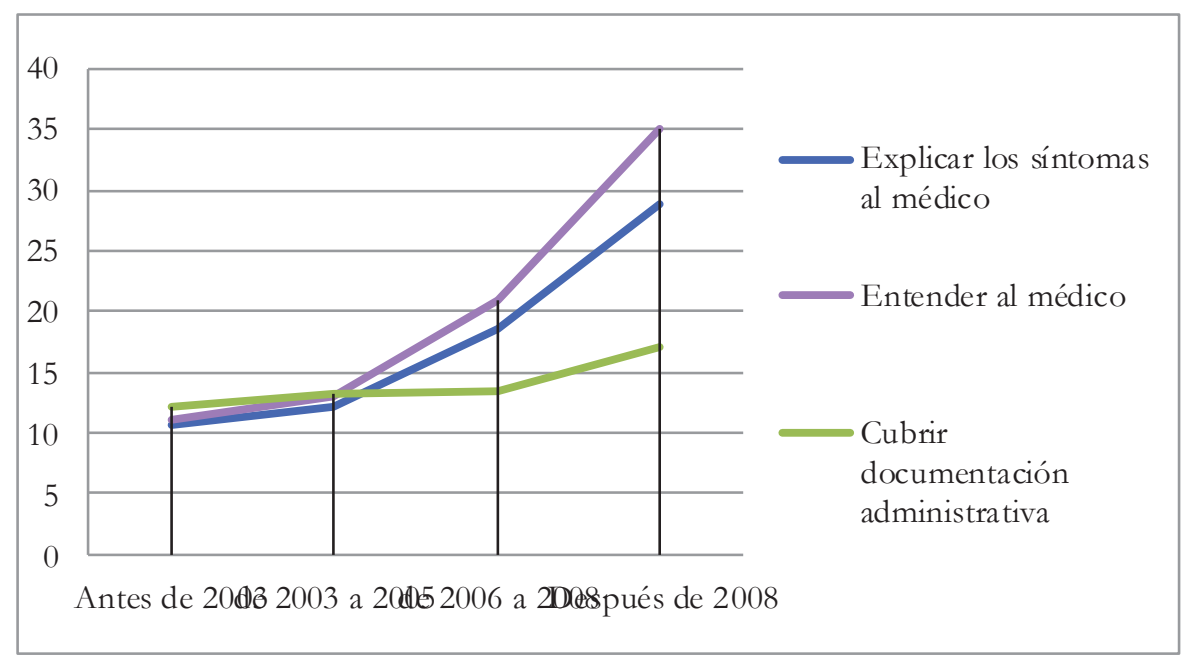

\section{Gráfico 4. Problemas durante la interacción médica según el tiempo de estancia}

\subsection{EL FACTOR DEL TURISMO EXTRANJERO EN ITALIA}

Como hemos señalado al principio del artículo, el servicio sanitario se extiende también a los extranjeros no residentes, que identificamos, por ejemplo, en los turistas. Según los más recientes datos del ISTAT (2014b), que en Italia estos visitantes proceden sobre todo de Alemania, Francia y Reino Unido por lo que atañe a los países de la UE. Con respecto a los turistas no comunitarios, Suiza y Rusia resultan los principales países de procedencia dentro del área europea, y EE. UU., Japón, China y Australia las primeras naciones a nivel extraeuropeo. Estos datos presentan un panorama muy distinto del anterior, ya que las naciones que destacan resultan más estables a nivel económico y con combinaciones lingüísticas más comunes desde el ámbito de la formación académica de intérpretes. De hecho, a excepción de 
China, la clasificación que se propone no comparte ningún valor con los datos obtenidos sobre los extranjeros residentes. En detalle, a nivel lingüístico destacamos una fuerte disparidad entre los principales idiomas entre los extranjeros residentes y turistas, ya que el rumano el albanés y el marroquí se contraponen al alemán, el francés y el inglés.
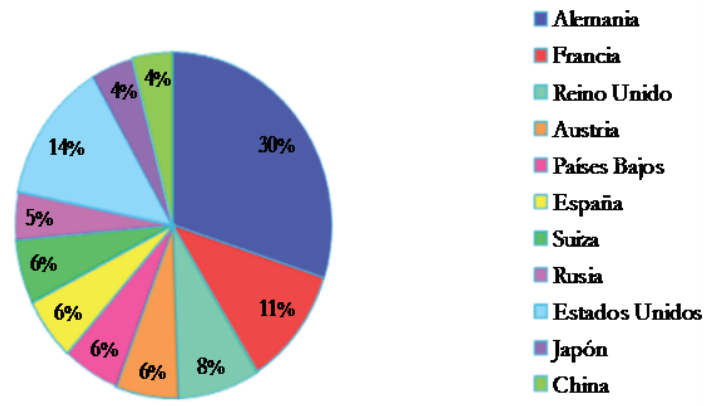

\section{Gráfico 5. Principales nacionalidades de turistas en Italia}

Sin embargo, si por un lado hemos destacado una marcada diferencia lingüística entre los dos grupos, por otro observamos una analogía en la elección del área geográfica, ya que, igual que los extranjeros residentes, los turistas privilegian el norte de Italia, al que sigue el centro $(30,1 \%)$ y por último el sur con el $12,3 \%$.

\section{ESTUDIO DE POBLACIÓN: LOS EXTRANJEROS EN ESPAÑA}

Al igual que Italia, España tiene una tradición de emigración que remota a los 70-80 primeros años del pasado siglo (ISTAT, 2014b). Aunque presenta una historia inmigratoria muy joven entre los países de la UE, en 2012 resultó ser el quinto Estado miembro con el mayor número de extranjeros por habitante.

Los datos obtenidos del INE (2014) sobre la población extranjera residente, relevan que también en España el primer país de procedencia de los inmigrantes es Rumanía (15,62\%); en segunda posición se encuentra Marruecos con el 15,27\%, otro estado que también hemos encontrado en la clasificación sobre Italia. Junto a Reino Unido (6,67\%), Ecuador (4,55 \%) e Italia $(3,90 \%$ ) estos cinco países representan el $46 \%$ de la población extranjera 
total en España. Como se puede observar de la siguiente tabla, Italia ha sido la única población que ha crecido durante el bienio 2013-2014, superando a Colombia, que en 2013 resultaba el quinto país más presente en el territorio español.

\begin{tabular}{|l|r|r|}
\hline \multicolumn{1}{|c|}{ País de procedencia } & 2013 & 2014 \\
\hline Rumanía & 769608 & 730340 \\
\hline Marruecos & 759273 & 714221 \\
\hline Reino Unido & 316362 & 311774 \\
\hline Ecuador & 269436 & 212970 \\
\hline Italia & 181046 & 182249 \\
\hline Colombia & 223140 & 172368 \\
\hline China & 169645 & 164555 \\
\hline Alemania & 153432 & 149522 \\
\hline Bulgaria & 147310 & 140206 \\
\hline Bolivia & 162538 & 126421 \\
\hline Portugal & 116431 & 109568 \\
\hline Francia & 101466 & 100448 \\
\hline Perú & 109639 & 83583 \\
\hline Ucrania & 84081 & 81625 \\
\hline Argentina & 95415 & 80910 \\
\hline Total de extranjeros & $\mathbf{5 0 7 2 6 8 0}$ & $\mathbf{4 6 7 6} \mathbf{0 2 2}$ \\
\hline
\end{tabular}

Tabla 4. Principales nacionalidades extranjeras en España en 2013 y 2014

Con respecto a la distribución de los extranjeros en el territorio, el INE releva Cataluña como primera comunidad autónoma por número de inmigrantes (que corresponden a 1.103.073), a la que siguen la Comunidad de Madrid con 810.425, Andalucía (665.093) y la Comunidad Valenciana (659.237). Parece preciso observar que no solo estas cuatro comunidades acogen el 65,49\% de los inmigrantes residentes totales, sino también que el $50,47 \%$ de estos extranjeros se reparten entre seis provincias españolas: Madrid, Barcelona, Alicante, Valencia, Málaga e Illes Baleares (OPI, 2014: 8). 


\subsection{PRINCIPALES MOTIVOS DE INMIGRACIÓN A ESPAÑA}

En el informe nacional sobre la inmigración y el trabajo, Carrasco y García Serrano (2012: 39) indican que los inmigrantes piden residencia en España principalmente por razones familiares, laborales y de estudio. En el siguiente gráfico se enseña la variación que se ha registrado durante el bienio 2009-2010 y los motivos de residencia en la península hispánica. Aunque los datos se remonten a hace cinco años, nos parece necesario destacar la versatilidad de estos valores.

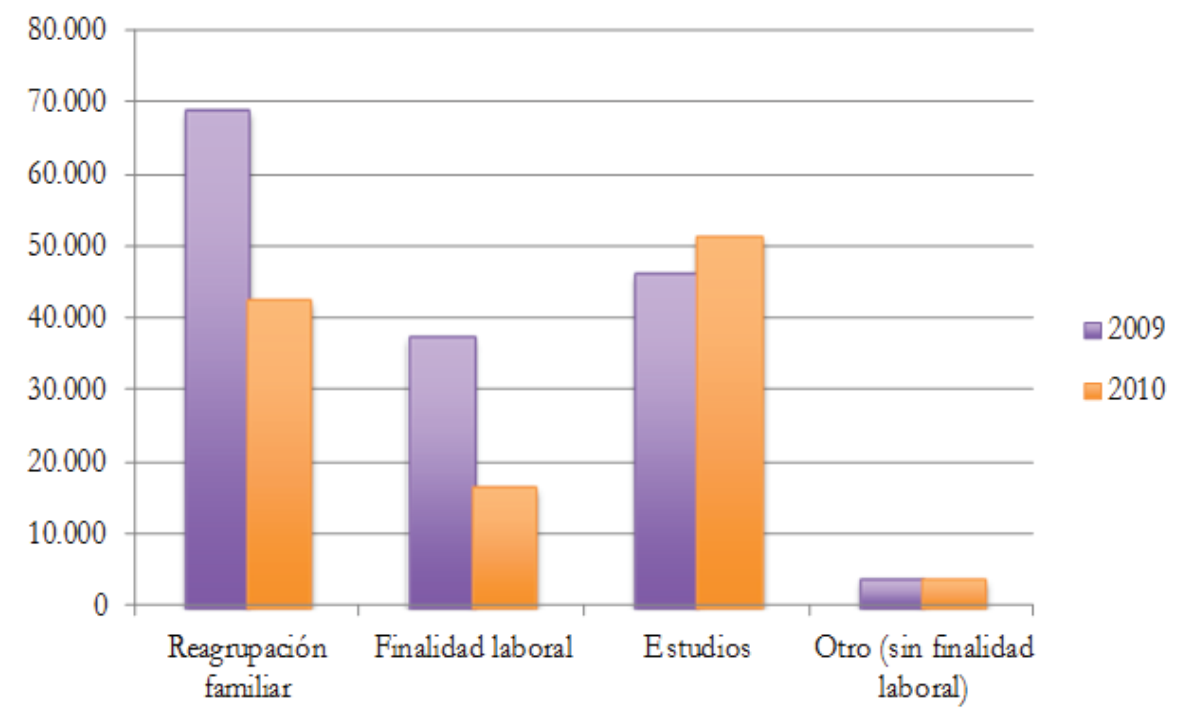

Gráfico 6. Principales motivos de residencia en 2009 y 2010

Otra información relativa a los motivos de estancia se encuentra en el informe Extranjeros residentes en España a 31 de diciembre de 2013, redactado de 2014 por el Observatorio Permanente de la Inmigración (OPI), sobre las razones de residencia temporal. Por un lado, encontramos datos sobre los ciudadanos con Régimen Comunitario, o sea población extranjera que procede de países de la UE y de la AELC7; de este primer grupo, el 68,17\% no ha determinado el motivo de residencia, valor que se justifica porque los certificados de registro expedidos resultan antecedentes a la entrada en vigor de la reforma del RD 240/2007 sobre la regulación de la residencia en España. Relevante es también el grupo compuesto por los ciudadanos con razones de 
residencia permanente $(25,17 \%)$, al que siguen los inmigrantes que piden residencia por trabajo por cuenta ajena $(3,34 \%)$, de residencia no lucrativa $(1,31 \%)$, por razones familiares $(1,01 \%)$, por estudio $(0,73 \%)$ y por trabajo por cuenta propia que corresponden al 0,27\% de los ciudadanos (2014: 9). Por otro lado, entre los extranjeros con Régimen General8 244321 tienen autorización de residencia temporal por trabajo por cuenta ajena, 187.042 por reagrupación familiar, 74250 por residencia no lucrativa y 44022 por arraigo. En menor medida estaría el trabajo por cuenta propia, otras autorizaciones de trabajo y las razones humanitarias (2014: 15).

\subsection{LOS EXTRANJEROS Y LOS SERVICIOS SANITARIOS}

Por lo que atañe al tema de la sanidad en España, en el marco legal se afirma que los residentes en España gozan de un alto nivel de acceso a los servicios de sanidad pública, lo que lleva a entender que hay una cobertura uniforme entre la población en España, aunque proceda de otros países o tenga otra situación administrativa (Blanco y Thuissard 2010: 97). En su estudio sobre la inmigración y la sanidad pública en España, Rafael Muñoz de Bustillo y José-Ignacio Antón afirman que la falta o el pobre conocimiento de la lengua puede constituir uno de los factores que hacen que los extranjeros utilicen menos los servicios de salud, puesto que puede representar un serio obstáculo en la comunicación con el personal sanitario (2009: 488).

Los datos sobre la asistencia sanitaria en España nos enseñan que entre 2011 y 2012 el 28,34 \% de los extranjeros utilizó los servicios de urgencias en el último año y que la mayoría eran personas entre los 25 y los 64 años. Si comparamos estas cifras con los datos relativos a la población española observamos que el servicio de urgencias ha sido utilizado en igual medida por el 28,31\%, de los cuales la mitad eran ciudadanos con edades comprendidas entre los 25 y los 64 años. Sin embargo, mientras que los inmigrantes entre los 15 y los 24 años ocupan la segunda posición con el 15,62\%, entre los españoles se encuentran los mayores de 65 años con un porcentaje del 19,36 \%.

Los valores varían un poco más con respecto al motivo de la consulta. De hecho, aunque tanto ambos grupos prefieran la Sanidad Pública, entre los españoles se observa una incidencia del $88 \%$ frente al $92,4 \%$ de los inmigrantes; sigue la consulta privada con el 6,5\% y el 4,5\% respectivamente, y la sociedad médica con unos valores que oscilan entre el 4,1\% de los nativos y el 1,7\% de los extranjeros. En el caso del médico de familia o del pediatra, el

${ }^{8}$ El Régimen General se aplica a los extranjeros "que no sean nacionales de los Estados miembros de la Unión Europea, del Espacio Económico Europeo y de la confederación Suiza” (ATA, en línea). 
porcentaje sigue mayor entre los españoles, con el 29,4\% frente al 24,3\% de los inmigrantes; lo mismo se observa en la consulta del médico especialista, donde se registran el $15 \%$ y el 10,7 \% respectivamente.

A diferencia del estudio sobre Italia, en el caso de España no se han encontrado datos sobre las dificultades de comunicación entre los inmigrantes y los servicios sanitarios, impidiendo una comparación lingüística más detallada entre las dos realidades.

\subsection{EL TURISMO EXTRANJERO EN ESPAÑA}

Destino anhelado por su clima mediterráneo y su cultura, también España acoge un número incesante de turistas. Igual que Italia, las tres naciones de procedencia de los de procedencia resultan ser Reino Unido, Alemania y Francia, a los que siguen los Países Nórdicos e Italia.

\begin{tabular}{|l|r|r|}
\hline País de procedencia & cantidad & porcentaje \\
\hline Reino Unido & 13654 & $23,7 \%$ \\
\hline Alemania & 9336 & $16,2 \%$ \\
\hline Francia & 8969 & $15,5 \%$ \\
\hline Países Nórdicos & 4165 & $7,2 \%$ \\
\hline Italia & 3572 & $6,2 \%$ \\
\hline Resto de Europa & 2782 & $4,8 \%$ \\
\hline Países Bajos & 2549 & $4,4 \%$ \\
\hline Resto del mundo & 2132 & $3,7 \%$ \\
\hline Resto de América & 1890 & $3,3 \%$ \\
\hline Portugal & 1861 & $3,2 \%$ \\
\hline Bélgica & 1706 & $3,0 \%$ \\
\hline Suiza & 1449 & $2,5 \%$ \\
\hline EE.UU. & 1241 & $2,2 \%$ \\
\hline Rusia & 1206 & $2,1 \%$ \\
\hline Irlanda & 1190 & $2,1 \%$ \\
\hline TÓTAL & 57701 & $\mathbf{1 0 0 , 0} \%$ \\
\hline
\end{tabular}

Tabla 5. Principales nacionalidades de turistas en España en 2012

Aunque en menor medida, también para España observamos una variación entre los principales idiomas entre las poblaciones inmigrantes y turísticas. Por lo tanto, mientras que los extranjeros residentes resultan hablar principalmente el rumano, el árabe, el inglés, el italiano y el chino, los turistas, a 
excepción de los británicos y los italianos, son sobre todo de habla alemana, francesa y de idiomas nórdicos.

Como en el caso de Italia, también en España se observa una equivalencia entre las áreas geográficas elegidas por los inmigrantes y los turistas. A excepción de las Canarias, también en ámbito turístico las cinco comunidades más elegidas resultan ser Cataluña, las Baleares, Andalucía, la Comunidad Valenciana y la Comunidad de Madrid (IET, en línea).

Finalmente, queremos destacar que entre las motivos que llevan a los turistas extranjeros a la tierra del toro, excepción hecha de la mayoría que viaja a España por ocio y vacaciones (86\%), el IET (2012: 28) revela que el $7 \%$ viaja por razones de trabajo y negocios, mientras que el 4,2 \% lo hace por motivos personales, motivos que incluyen, entre otros, las visitas a familiares y la salud, destacando así otro tipo de turismo: el turismo sanitario.

\section{ANÁLISIS COMPARATIVO DE LOS DATOS DE POBLACIÓN}

Si comparamos los datos poblacionales de los dos países, podemos realizar algunas interesantes reflexiones que nos permiten definir aun más el panorama que estamos estudiando.

Una primera observación atañe al número total de extranjeros residentes en los dos territorios. Desde un punto de vista cuantitativo, España presenta sin duda un número mayor de extranjeros con respecto a Italia (5 072680 frente a 4387 721). Sin embargo, si analizamos la situación desde el punto de vista lingüístico, observamos que las dos realidades se parecen mucho más de lo que aparentan, ya que, en el caso de España, solo eliminando los valores de los inmigrantes procedentes de Ecuador y Colombia, el número total español pasa a ser 4.580.104, acercándose mucho más al italiano.

Además, con respecto a las nacionalidades extranjeras presentes en

Italia y en España, Rumanía, Marruecos y China se encuentran entre las naciones más representativas en ambos países. 


\section{ITALIA}

\section{ESPAÑA}
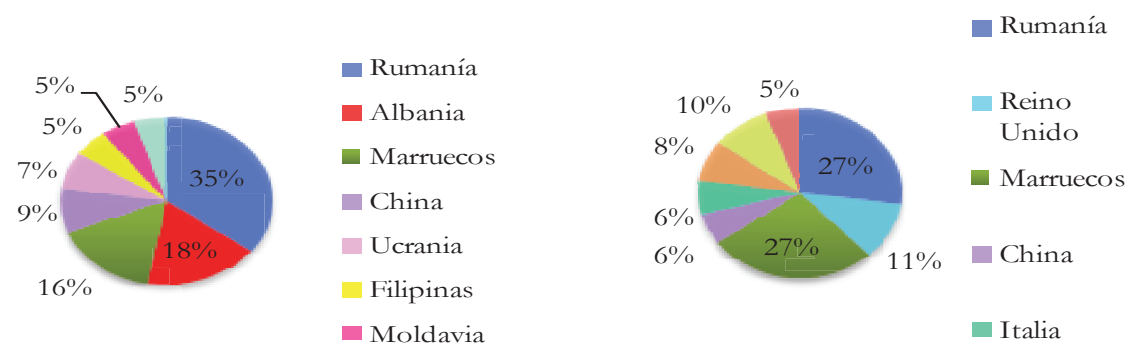

\section{Gráfico 7. Principales nacionalidades de residentes en Italia y España}

Desde el punto de vista de la interpretación sanitaria, aunque en el caso de España faltan los valores relativos a los problemas relativos a la interacción lingüística entre el paciente extranjero y el personal sanitario, podemos suponer que los resultados sean muy similares a los italianos, sobre todo porque se trata de dos lenguas románicas muy parecidas entre sí, que nos permiten extrapolar que las dificultades que se encuentren en la comunicación médico-paciente sean muy similares en los dos casos.

Hay que poner de relieve que, desde un punto de vista económico, las nacionalidades más presentes en Italia resultan más débiles que las españolas. De hecho, mientras que en el bel paese encontramos a Albania, Ucrania y Filipinas, en España los extranjeros residentes proceden, por orden de importancia relativa, de Reino Unido, Alemania e Italia. Además, desde un punto de vista traductológico, siendo el albanés, el ucraniano y el filipino idiomas atípicos dentro del mundo académico italiano, resulta mucho más complejo atender a este grupo de hablantes; de hecho, estas son lengua que casi solo dominan los hablantes nativos, lo que, a nivel de integración social y cultural acrecienta la situación de minorías étnicas de estas comunidades lingüísticas. En España, esta situación se reproduce mucho menos que en Italia, ya que el inglés, el alemán y el italiano son tres lenguas que no se limitan a su comunidad nativa, sino que se pueden aprender a partir de la escuela secundaria y para las cuales se proporciona una amplia oferta académica también en el ámbito de la traducción e interpretación. 
Para los datos sobre el turismo, se destaca un cambio radical en las nacionalidades que más viajan a las dos penínsulas europeas. De hecho, podemos observar cómo el factor económico está equilibrado en ambos países.

ITALIA

Alemania

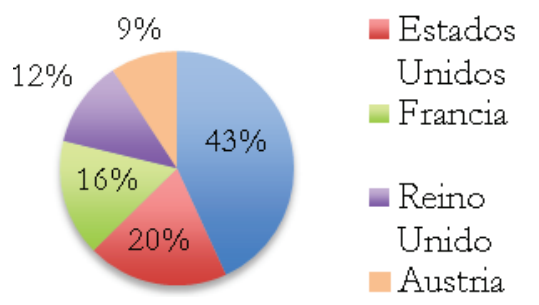

\section{ESPAÑA}

Alemania

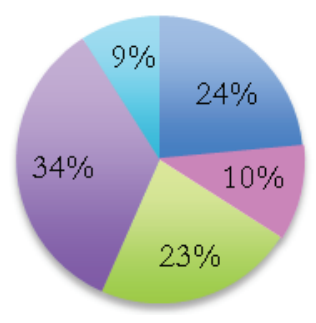

- Países

Nórdicos

Francia

- Reino

Unido

- Italia

\section{Gráfico 8. Principales nacionalidades de turistas en Italia y España}

En el caso de España, Alemania, Reino Unido e Italia mantienen una presencia importante también a nivel turístico. Desde el punto de vista lingüístico notamos que para Italia el abanico de idiomas cambia de manera radical. De hecho, se encuentran tres de los cuatro idiomas más conocidos por la población nacional (ISTAT, 2014d: 1), en concreto, distinguimos el alemán, el inglés y el francés. En cambio, España, presenta una diversificación lingüística mayor. Aparte de las lenguas nórdicas, también en este caso observamos cómo los idiomas más representativos son el inglés, el alemán, el francés y el italiano, que representan los cuatro idiomas más estudiadas en el país (Eurobarómetro 2012; MECD 2014: 12).

\section{CONCLUSIONES}

El estudio poblacional sobre los extranjeros residentes y turistas en Italia y España que se ha llevado a cabo, nos ha permitido llegar a los siguientes resultados:

1. El total de extranjeros presentes en ambos países es muy parecido, sobre todo si consideramos solo las nacionalidades que hablan un 
idioma distinto del país en examen. (4 580104 en España y 4387721 en Italia).

2. El fenómeno migratorio no se distribuye de manera homogénea sino de manera localizada en ambos países, privilegiando el norte para Italia (Tabla 6) y Cataluña, Comunidad de Madrid, Andalucía y Comunidad Valenciana para España (Tabla 7).

\begin{tabular}{|l|r|r|}
\hline \multicolumn{1}{c|}{ Regiones } & Italianos & Extranjeros \\
\hline Lombardía & 8756863 & 947288 \\
\hline Véneto & 4399882 & 457328 \\
\hline Emilia-Romaña & 3890099 & 452036 \\
\hline Lacio & 5077179 & 425707 \\
\hline Piamonte & 4004568 & 359348 \\
\hline
\end{tabular}

Tabla 6. Distribución poblacional en Italia por regiones, año $2011^{9}$

\begin{tabular}{|l|r|r|}
\hline \multicolumn{1}{|c|}{ Comunidades autónomas } & Españoles & Extranjeros \\
\hline Cataluña & 7480921 & 1103073 \\
\hline Comunidad de Madrid & 6414709 & 810425 \\
\hline Andalucía & 8393159 & 665093 \\
\hline Comunidad Valenciana & 4987017 & 659237 \\
\hline
\end{tabular}

Tabla 7. Distribución poblacional en España por comunidades autónomas, año 2013

3. Las razones que llevan a la migración son sobre todo familiares y laborales. Además, con respecto al tema laboral en Italia, los trabajadores extranjeros son sobre todo obreros y artesanos o no cualificados, de modo que llegamos a la conclusión que pertenecen sobre todo a un estatus social medio-bajo.

${ }^{9}$ Datos ISTAT del 24 de septiembre de 2014. 
4. Entre las nacionalidades más representantes de los dos países, encontramos Rumanía Marruecos y China presentes en ambos territorios.

5. Se nota una diferencia económica entre los principales grupos de extranjeros en Italia (Albania, Ucrania y Filipinas) y en España (Reino Unido, Alemania e Italia).

6. También desde un punto de vista traductológico los extranjeros de Italia resultan más discriminados, ya que resulta más difícil atender a hablantes de albanés, ucraniano y filipino que a hablantes de inglés, alemán e italiano, siendo lenguas que dentro del mundo académico prácticamente no existentes.

7. Para Italia la situación lingüística y económica cambia radicalmente con respecto a los datos sobre el turismo, mientras que España registra una mayor coherencia, ya que se encuentran Alemania, Reino Unido e Italia entre los principales países de procedencia también entre los valores turísticos.

A pesar de la diferencia lingüística presentes entre los dos grupos de extranjeros analizados, hay distintos factores que coinciden en Italia y España. En concreto, destacamos los siguientes puntos:

1. Tanto Italia como España han sido primero países de emigración.

2. Ambas son naciones con una tradición inmigratoria joven.

3. Los dos países resultan muy parecidos geográfica y culturalmente; de hecho, ambos son Estados miembros del sur de Europa, ofrecen un clima mediterráneo y un bagaje cultural y culinario que atrae a millones de turistas durante todo el año.

4. Desde un punto de vista económico, proporcionan dos realidades muy parecidas. En detalle, si por un lado Italia ofrece una oferta laboral un poco mejor que España, por otro lado esta última lo compensa con un coste de vida más bajo.

5. En el plan lingüístico, el italiano y el español son idiomas muy parecidos, con dificultades de aprendizaje similares.

6. Con respecto a los servicios sanitarios, aunque tenemos datos relativos a los problemas lingüísticos solo en el caso de Italia, podemos suponer que la situación puede ser muy similar también en España, dada la similitud de los movimientos migratorios y de las dos lenguas.

Estos resultados nos han permitido constatar la importancia que la confluencia de lenguas y culturas tiene tanto en Italia como en España. De hecho, hemos podido demostrar una vez más la necesidad para ambos países 
de ofrecer un servicio de interpretación estable y homogéneo, con el fin de proporcionar una asistencia equitativa y de calidad también a las minorías étnicas. No tenemos que olvidar que ambas naciones hemos sido primero emigrantes, viviendo en primera persona la situación de disparidad por ser una minoría lingüística y cultural en otras latitudes.

Finalmente, esperamos que lo aquí demostrado sirva como punto de partida para que las instituciones y las universidades empiecen a colaborar y a proporcionar una demanda y una oferta profesional coherente en estas dos realidades, sobre todo si consideramos que tanto Italia como España todavía carecen de regulación en el ámbito específico de la interpretación sanitaria. De hecho, solo si encontramos un punto común, también a escala internacional, podremos lograr el reconocimiento y la regulación a nivel nacional que la interpretación comunitaria y sus especialidades merecen.

\section{REFERENCIAS BIBLIOGRÁFICAS}

ATA Federación Autónomos. "Régimen General" en La ley. Documento electrónico disponible en: http://www.autonomosinmigrantes.com/pdf/laley/regimenGeneral.pdf.

Blanco Moreno, Ángela Israel y Thuissard Vasallo, John (2010). "Gasto sanitario e inmigración: una mirada en clave de integración” en Presupuestos y Gasto Público, 61, pp. 93-132.

Carrasco Carpio, Concepción y García Serrano, Carlos (2012). Inmigración y mercado de trabajo. Informe 2011. Madrid, Ministerio de Empleo y Seguridad Social.

Centro Studi CNA (2013). L'imprenditoria straniera in Italia nel 2012. Roma. CNA. Comisión Europea (2012). Special Eurobarometer 386. Europeans and their Languages. Report. Bruselas. Comisión Europea.

- (2006). Standard Eurobarometer 66. Public Opinion in the European Union. Bruselas, Comisión Europea.

Dumont, Jean-Christophe, Spielvogel, Gilles y Widmaier, Sarah (2010). "International Migrants in Developed, Emerging and Developing Countries: An Extended Profile en OECD Social Employment and migration Working Papers, n. ${ }^{\circ}$ 114. París. Organización para la Cooperación y el Desarrollo Económicos (OCDE).

EUROSTAT. Oficina de estadística de la Comisión europea. En línea: http://epp.eurostat.ec.europa.eu/portal/page/portal/eurostat/home/.

Falvo, Maria (2010). L'interprete di tribunale: Italia e Spagna a confronto. Tesi di Laurea. Università di Bologna. 
IET. Instituto de Estudios Turísticos. En línea: http://www.iet.tourspain.es/enEN/Paginas/default.aspx.

IET (2012). Informe anual 2012. Movimientos Turísticos en Fronteras (Frontur). Encuesta de Gasto Turístico (Egatur). Madrid, Ministerio de Industria, Energía y Turismo.

INE. Instituto Nacional de Estadística. En línea: http:/ /www.ine.es/.

INE (2014). Notas de prensa. Cifras de Población a 1 de enero de 2014. Estadística de Migraciones 2013. Datos Provisionales.

ISTAT. Istituto nazionale di statistica. En línea: http://www.istat.it/it/.

- (2013). La classifica delle professioni. Roma. Stealth.

- (2014). Anno 2011-2012. Cittadini stranieri: condizioni di salute, fattori di rischio, ricorso alle cure e accessibilità dei servizi sanitari.

- (2014a). Anno 2011-2012. Cittadini stranieri: condizioni di salute, fattori di rischio, ricorso alle cure e accessibilità dei servizi sanitari. Tavole.

- (2014b). Annuario statistico italiano 2013. Roma. Rubbettino print.

- (2014c). Report. Cittadini non comunitari regolarmente soggornanti.

- (2014d). Report. L'uso della lingua italiana. Dei dialetti e di altre lingue in Italia. Anno 2012.

Ministerio de Educación, Cultura y Deporte - MECD (2014). Estadística de las enseñanzas no universitarias - curso 2011-2012.

Ministero del Lavoro e delle Politiche Sociali (2014). Quarto rapporto annuale. Gli immigrati nel mercato del lavoro in Italia. Roma, Direzione Generale dell'Immigrazione e delle politiche di integrazione.

Muñoz de Bustillo, Rafael y Antón, José-Ignacio (2009). "Health care utilisation and immigration in Spain" en European Journal of Health Economics (2010), 11, pp. 487-498.

OECD. The Organization for Economic Co-operation and Development. En línea: http://www.oecd.org/.

OECD (2014a). International Migration Outlook 2013. Paris, OECD Publishing.

- (2014b). Jobs for Immigrants (Vol. 4). Labour Market Integration in Italy. Paris. OECD Publishing.

OIM (2013). Informe sobre las Migraciones en el Mundo 2013: El Bienestar de los Migrantes y el Desarrollo. Ginebra. Organización Internacional para las Migraciones.

OPI (2014). Extranjeros residentes en España a 31 de diciembre de 2013. Principales resultados. Madrid. Ministerio del Interior.

Zanin, Valter y Mattiazzi, Giulio, coords. (2011). Migrazione, lavoro, impresa. Tra America Latina en Europa. Torino, L'Harmattan Italia. 